\title{
Artificially layer heavy Fermion superconductors - a new opportunity to probe unconventional superconductivity
}

Extremely strong-coupling superconductivity in artificial two-dimensional Kondo lattices Authors: Y. Mizukami, H. Shishido, T. Shibauchi, M. Shimozawa, S. Yasumoto, D. Watanabe, M. Yamashita, H. Ikeda, T. Terashima, H. Kontani and Y. Matsuda

Nature Physics 7 online: http://www.nature.com/nphys/journal/vaop/ncurrent/full/nphys2112.html (October 9, 2011)

\section{Recommended and a Commentary by Manfred Sigrist, ETH Zurich, Switzerland}

CeCoIn 5 belongs to the group of Ce-115 heavy Fermion superconductors which are intimately connected with antiferromagnetic order and strongly enhanced spin fluctuations which likely takes a major part in forming unconventional Cooper pairs with $d$-wave symmetry $[1,2]$. This superconductor has received considerable attention in recent years, after a new phase was observed at very low temperatures and high magnetic fields, which is widely viewed as a strong candidate for a Fulde-Ferrel-Larkin-Ovchinnikov phase [3, 4]. A further twist to this story came with the discovery of a incommensurable spin density wave state coexisting with this superconducting phase, known nowadays as the "Q-phase" [5, 6].

In their very recent article Mizukami et al report the successful fabrication of an artificial layered superstructure $\mathrm{CeCoIn}_{5}$ and $\mathrm{YbCoIn}_{5}$. While each Ce-atom contributes one 4f-electron to a regular Kondo lattice, $\mathrm{Yb}$ enters with a completely filled $4 f$-shell $\left(4 f^{14}\right)$ such that the $\mathrm{YbCoIn}_{5}$ layers are simply metallic without forming heavy quasiparticles. Therefore we may expect to see here a layered structure with alternating conventional and heavy-fermion metal property. The Ce-115 superconductors were synthesized originally as variants of genuinely 3-dimensional heavy fermion superconductor $\mathrm{CeIn}_{3}$ to investigate the influence of reduced dimensionality on superconductivity. Indeed $\mathrm{CeCoIn}_{5}$ has a considerably larger $T_{c}=2.3 \mathrm{~K}$ than $\mathrm{CeIn}_{3}$ which becomes superconducting only under pressure. It is among the highest $T_{c} \mathrm{~s}$ in the Ce-class. However, despite the $\mathrm{CoIn}_{2}$ spacer layer in the crystal structure, the two-dimensionality is not very pronounced in its normal and superconducting properties. Consequently, the availability of an artificially layered structure in which the number of CeCoIn 5 layers can be varied, being separated by several layers of $\mathrm{YbCoIn}_{5}$, is most welcome for a systematic study of effects due to dimensional reduction. Mizukami et al choose $5 \mathrm{YbCoIn}_{5}$ layers as spacer in order to reduce magnetic coupling (RKKY coupling) among the Ce-blocks strongly. Thus, they report results for $(n, 5)$ superlattices, $n$ being the number of CeCoIn-5 layers.

Most amazingly superconductivity survives in these artificial structures. First of all this is a clear sign of the high sample quality, since unconventional pairing - in this case presumably $d_{x^{2}-y^{2} \text {-wave }}$ pairing - is rather easily suppressed by disorder. However, most surprising is the fact that even for $n=1$ and 2 still resistivity shows signatures of superconductivity and that already for $n \geq 3$ the resistivity drops to zero at finite temperatures. The reduction of $T_{c}$ is not so surprising in view of the fact that the Ce-layer blocks are sandwiched between the metallic spacer $\mathrm{YbCoIn}_{5}$. These metallic layer yield a suppression of the superconducting order parameter through proximity effect, 
the leaking of a Cooper pairs from the Ce-blocks to the Yb-blocks.

An important question is the nature of the quasiparticles making superconductivity. Heavy electron features are still faintly observable through the "coherence peak" in resistivity around 20 to $50 \mathrm{~K}$. What is measured in transport is, however, a complex multi-component signal. Most convincing for the heavy electron character of the superconducting quasiparticles is the short coherence length (indicating "slow electrons") derived from the very high upper critical field. Indeed the upper critical field stays as high as in the bulk material, although the critical temperature decreases with shrinking layer number $n$. Taking the rough estimate $\xi \sim \hbar v_{F} / k_{B} T_{c}$ we must conclude that the quasiparticles are slow, i.e. heavy.

The two-dimensionality of this new superconducting system is visible in the diverging ratio $H_{c 2 \|} / H_{c 2 \perp}$ for $T \rightarrow T_{c}$. While the upper critical field perpendicular to the layers follows a standard linear $T$ dependence, $H_{c 2 \perp} \propto\left|T-T_{c}\right|$, a sub-linear behavior is expected for inplane fields, i.e. $H_{c 2 \|} \propto\left|T-T_{c}\right|^{\alpha}$ with $\alpha<1$. This is indeed beautifully observed in the measurements.

In the title "strong-coupling superconductivity" is invoked for these layered compound. The argument is based on the analysis of the upper critical field. As for the $\mathrm{CeCoIn}_{5}$ bulk system the low-temperature upper critical field is likely given by paramagnetic limiting, i.e. the depairing through spin polarization of the spin-singlet Cooper pairs. Indeed the a comparison of the zerotemperature limit with the slopes of $H_{c 2}$ at the onset of superconductivity suggests that for all field directions paramagnetic limiting is the likely cause of depairing in a field. This prompts Mizukami et al to adopt the standard relation

$$
H_{c 2}^{(\text {para })} \approx \frac{\sqrt{2} \Delta}{g \mu_{B}} .
$$

From the enhanced values of $H_{c 2} / T_{c}$, they conclude that the ratio $\Delta / k_{B} T_{c}$ takes values as high as 10 for the layered structure, considerably larger than the already large bulk value of 6 . This remarkable enhancement they take as evidence for increased strong-coupling effects. In my view this

conclusion has to be taken with some caution. First of all the simple equation for $H_{c 2}^{(\text {para })}$ is valid only on a rather approximative level. Paramagnetic limiting is connected with the reduction of spin polarizability in the superconducting state. Therefore we may also ask whether the enhanced $H_{c 2}$ could have different origins than a strongly enhanced condensation energy. One possibility has been proposed by Murakami et al [7], considering the possible spin-orbit effects caused by the crystal structure of the artificially layered system, in particular, due to locally removed inversion symmetry which can reduce paramagnetic limiting effects. Therefore the conclusion that the unexpectedly high $H_{c 2}$ is a consequence of strong coupling is not necessarily a unique conclusions.

I believe that the study by Mizukami and collaborators marks a truly astounding advance which is pioneering development for strongly correlated electron systems including heavy Fermion compounds towards a new era of design of rather complex materials classes.

\section{References}

[1] J.L. Sarrao and J.D. Thompson, J. Phys. Soc. Jpn 76, 051013 (2007).

[2] C. Pfleiderer, Rev. Mod. Phys. 81, 15511624 (2009). 
[3] A. Bianchi, R. Movshovich, C. Capan, P.G. Pagliuso and J.L. Sarrao, Phys. Rev. Lett. 91, 187004 (2003).

[4] Y. Matsuda and H. Shimahara, J. Phys. Soc. Jpn. 76051005 (2007).

[5] B.-L. Young, R. R. Urbano, N. J. Curro, J. D. Thompson, J. L. Sarrao, A. B. Vorontsov, and M. J. Graf, Phys. Rev. Lett. 98, 036402 (2007).

[6] M. Kenzelmann et al, Science 321, 1652 (2008).

[7] D. Murakami, Y. Yanase and M. Sigrist, cond-mat/1110.6000 